\title{
Parametrically excited MEMS vibration energy harvesters with design approaches to overcome the initiation threshold amplitude
}

\author{
Yu Jia, Jize Yan, Kenichi Soga and Ashwin A Seshia \\ Department of Engineering, University of Cambridge, Trumpington Street, \\ Cambridge CB2 1PZ, UK \\ E-mail: yj252@cam.ac.uk, jy242@cam.ac.uk, ks207@cam.ac.uk, \\ aas41@cam.ac.uk
}

\begin{abstract}
.
Resonant-based vibration harvesters have conventionally relied upon accessing the fundamental mode of directly excited resonance to maximise the conversion efficiency of mechanical-to-electrical power transduction. This paper explores the use of parametric resonance, which unlike the former, the resonant induced amplitude growth is not limited by linear damping and wherein can potentially offer higher and broader nonlinear peaks. A numerical model has been constructed to demonstrate the potential improvements over the convention. Despite the promising potential, a damping-dependent initiation threshold amplitude has to be attained prior to accessing this alternative resonant phenomenon. Design approaches have been explored to passively reduce this initiation threshold. Further, three representative MEMS designs were fabricated with both $25 \mu \mathrm{m}$ and $10 \mu \mathrm{m}$ thick device silicon. The devices include electrostatic cantileverbased harvesters, with and without the additional design modification to overcome initiation threshold amplitude. The optimum performance was recorded for the $25 \mu \mathrm{m}$ thick threshold-aided MEMS prototype with device volume $\sim 0.147 \mathrm{~mm}^{3}$. When driven at $4.2 \mathrm{~ms}^{-2}$, this prototype demonstrated a peak power output of $10.7 \mathrm{nW}$ at the fundamental mode of resonance and $156 \mathrm{nW}$ at the principal parametric resonance; as well as a 23 fold decrease in initiation threshold over the purely parametric prototype. An approximate doubling of the half power bandwidth was also observed for the parametrically excited scenario.
\end{abstract}

PACS numbers: 07.07.Mp, 07.10.Cm, 07.07.Df

Submitted to: J. Micromech. Microeng.

\section{Introduction}

In recent years, there has been an increased interest in vibration energy harvesting, especially to enable self-powered wireless sensor networks for structural health monitoring [1]. Additionally, MEMS technology is viewed as a vehicle to realise mass fabrication of vibration energy harvesters (VEH), similar to the economies of scales achieved from semiconductor batch manufacturing $[2,3,4]$.

The three most popular mechanical-to-electrical transduction mechanisms implemented for MEMS VEH are summarised below [3, 5]. 
- Electromagnetic: power output does not scale well with dimension due to diminishing coil turn density and fill factor at smaller scales using current micromachining technologies.

- Electrostatic: mature MEMS technology, can be readily fabricated and integrated with IC technology. However, the peak power density, both theoretical and reported in the literature, is an order of magnitude lower than its counterparts.

- Piezoelectric: scales well and offers high performance, especially PZT, but MEMS fabrication is less straightforward compared to electrostatics. AlN and $\mathrm{ZnO}$ based harvesters are now more practically realisable with existing fabrication technology.

While some early commercial solutions have witnessed a modest increase in deployment [4], two of the key technical limitations still persist; namely, the low power density relative to conventional power supply and the confined operational frequency bandwidth while faced with the wideband nature of real vibrations.

This paper attempts to address these issues through employing parametric resonance instead of the convention of accessing the fundamental mode of directly excited resonance. The parametric resonant phenomenon contains a self excitation component, which unlike the conventional approach, does not exhibit amplitude saturation due to linear damping and is almost always associated with vibrational nonlinearities soon after its onset. Therefore, theory predicts the potential for an order higher [6] power output for such devices given the right boundary conditions. Additionally, the onset of nonlinearity that eventually saturates its growth helps to moderately widen the operational frequency bandwidth. Despite these promising advantages, an early experimental investigation of a parametrically excited cantilever design [7] failed to report notable performance enhancements over the current paradigm. One of the key obstacles to introduce parametric resonance into a system is the presence of a damping-dependent initiation threshold amplitude, which excitation must overcome prior to accessing parametric resonance.

Following sections numerically and experimentally investigate the potential enhancements of parametric resonance over the fundamental mode of resonance, as well as exploring design approaches to overcome the limitation of initiation threshold amplitude in order to practically realise the promising potential of parametrically excited vibration energy harvesters.

\section{Theory}

\subsection{Parametric resonance}

The motivation for employing parametric resonance for vibrational energy harvesting can be summarised as,

- Maximises power output by mechanically amplifying displacement amplitude.

- Broadens operational frequency bandwidth from the nonlinear resonant peak.

This resonant phenomenon is characterised by the presence of a time-dependent modulation in one of its system parameters as described by the generic damped Mathieu equation [8] shown in equation 1.

$$
\ddot{x}+c \dot{x}+(\delta+2 \varepsilon \cos (2 t)) x=0
$$


where, $x$ is displacement, $c$ is damping, $t$ is time domain, $\varepsilon$ is a generic excitation amplitude parameter and $\delta$ is a generic natural frequency squared parameter.

Parametric excitation, unlike direct excitation, is usually orthogonal rather than parallel to the plane of displacement. In order to achieve parametric resonance, it can be shown that the excitation frequency $\omega$ needs be approximately $2 \omega_{0} / n$; where $\omega_{0}$ is the natural frequency of the resonator and $n$ is an integer defining the order of parametric resonance. Therefore, the first order (principal) parametric resonance can be attained when the excitation frequency is twice the natural frequency. Additionally, there is a prerequisite of a non-zero initial displacement in order to 'push' the system out of stable equilibrium.

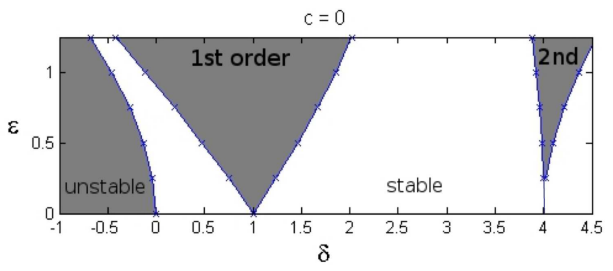

(a) Undamped

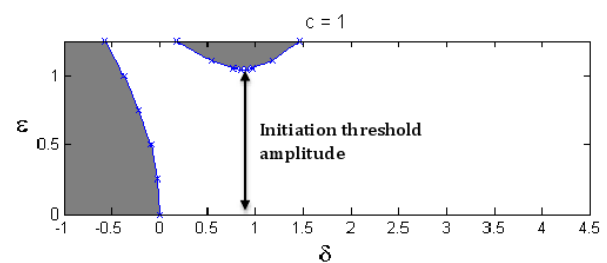

(b) Damped

Figure 1: $\varepsilon-\delta$ stability charts of the undamped and damped Mathieu equations. Shaded (unstable) regions are where parametric resonance is activated. With increasing damping $c$, higher excitation amplitudes are required to overcome the initiation threshold amplitude prior to accessing the unstable regions.

Apart from these requirements, the excitation amplitude needs to overcome a damping-dependent initiation threshold prior to accessing the parametric resonant region as illustrated in the $\varepsilon-\delta$ plane stability chart of Mathieu equation shown in figure 1. This appears counterproductive in the context of vibration energy harvesting, since the essence of the art relies on electrically damping the mechanical resonator in order to extract electrical energy. However, once parametric resonance is activated, the oscillatory amplitude growth is not limited by linear damping and can only be saturated by either physical limits or the onset of nonlinearity. This offers the potential to attain higher oscillatory amplitudes and power output.

\subsection{Cantilever resonators}

The cantilever resonator is a common design choice [4]. Large displacements are achievable at the free end (ideal for electromagnetic and electrostatic transducers), while simultaneously high levels of stress are induced near the clamped end (ideal for piezoelectric transducers) and offer lower natural frequencies than many other device configurations. Conventionally, the cantilevers are driven with direct excitation parallel to the displacement as summarised in equation 2.

$$
\ddot{x}+\frac{2 c_{1}}{m} \dot{x}+\frac{c_{2}}{m} \dot{x}|\dot{x}|+\frac{\mu}{m} x^{3}+\omega_{0}^{2} x=\omega^{2} A \cos (\omega t)
$$

where, $c_{1}$ is the linear damping parameter, $c_{2}$ is a quadratic damping representing the dominant higher order damping nonlinearity, $\mu$ is a parameter representing cubic geometric non-linearities, $A$ is the excitation displacement amplitude, $m$ is the effective 
mass, $\omega$ is the excitation frequency and $\omega_{0}$ is the angular natural frequency. The parameters $c_{1}, c_{2}$ and $\mu$ are normalised parameters against effective mass.

When a parametric excitation is applied perpendicular to the direction of the displacement, the equation of motion (a version of the damped Mathieu equation) shown in equation 3 can be observed [7].

$$
\ddot{x}+\frac{2 c_{1}}{m} \dot{x}+\frac{c_{2}}{m} \dot{x}|\dot{x}|+\frac{\mu}{m} x^{3}+\left(\omega_{0}^{2}-\frac{\omega^{2} A}{l} \cos (\omega t)\right) x=0
$$

where, $l$ is the effective beam length. Figure 2 numerically (using ode45 solver in MATLAB with 'Relative tolerance' of 1E-05) contrasts the resonant peaks of a microcantilever when subject to either types of excitation at varying acceleration levels. While direct excitation always yields a response regardless of the excitation level, its parametric counterpart converges to a zero steady-state response below the initiation threshold amplitude and a small non-zero initial displacement condition is required for any response to be possible. However, with increasing excitation amplitudes beyond this threshold barrier, parametric resonance rapidly outperforms the fundamental mode of resonance.

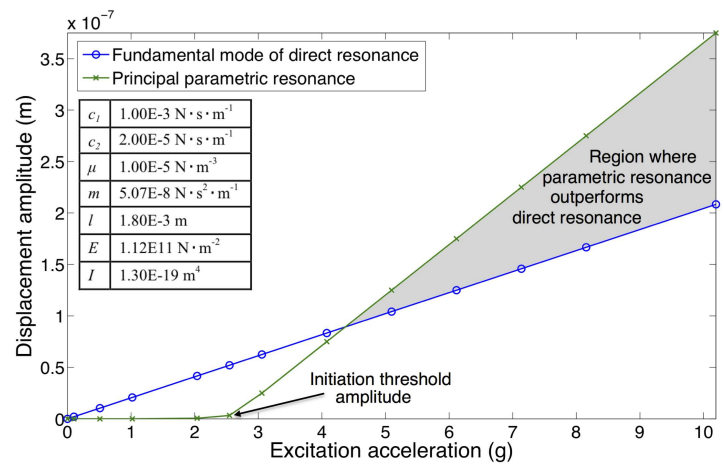

Figure 2: Numerical comparison of the steady-state resonant peaks between direct and parametric excitations at various excitation acceleration levels. $E$ and $I$ are the Young's modulus and second moment of area respectively.

Additionally, oscillatory build up typically undergoes a long transient state prior to converging to a steady state value compared to the directly excited resonant response (see figure 3). Nonetheless, it is able to eventually attain a higher response amplitude when operating within the shaded region from figure 2 .

Both this numerical simulation and an existing experimental study in the literature [7] have ascribed large values for this initiation threshold amplitude; whereas in practice, the ambient vibration available for harvesting is usually very small. Therefore, this initiation threshold amplitude must be minimised in order to practically exploit the merits of parametric resonance.

\section{Design and fabrication}

\subsection{Design paths}

Other applications, such as MEMS gyroscopes [9] have already attempted to employ parametric resonance as a means of mechanical amplification, typically incorporating 


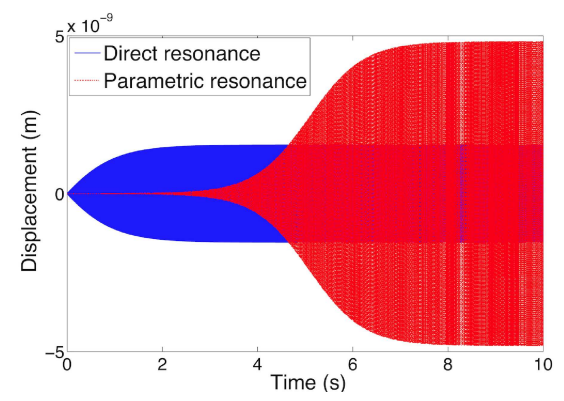

Figure 3: Oscillatory build up of parametric resonance usually takes longer but attains higher amplitudes soon beyond the initiation threshold amplitude. The applied amplitude is the same for these particular solutions.

active excitation to overcome the limitation of initiation threshold amplitude in order to access the parametric resonant regimes. However, the art of energy harvesting cannot afford the additional power expenditure. Therefore, a passive solution to fulfil this critical boundary condition is needed.

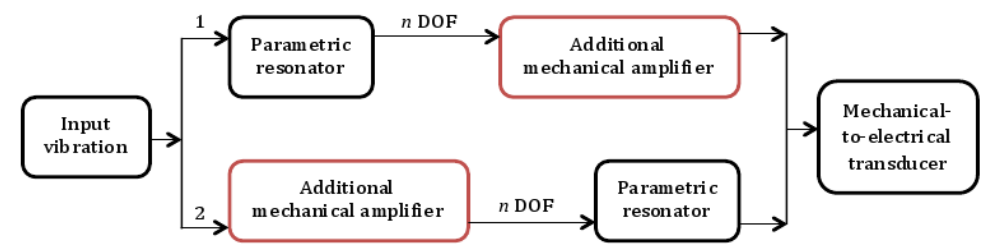

Figure 4: Design approaches to passively minimise initiation threshold amplitude of parametrically excited vibration energy harvesters. The term 'additional mechanical amplifiers' encompasses direct resonators, parametric resonators and non-resonating amplifiers such as levers; and ' $n$ DOF' stands for $n$ number of degrees-of-freedom.

Figure 4 presents the proposed design approaches in an attempt to address this issue. The first design route employs an electrically undamped parametric resonator that is coupled to an additional electrically damped mechanical amplifier. Here, the parametric resonator has intrinsically lower initiation threshold amplitude due to minimal damping. The second design route introduces an electrically undamped mechanical amplifier prior to the electrically damped parametric resonator. In this scenario, the base vibration amplitude is amplified and brought up towards the threshold. With reference to figure 1b, the first approach essentially lowers the position of the unstable region while the second approach raises the horizontal base axis.

This article shall explore experimental models based on the second design route from figure 4, while the first design route is investigated through another article by the authors [10]. A design model of a micro-cantilever with capacitive combs is shown in figure 5a. Out-of-plane vibration is equivalent to direct excitation while an inplane excitation force applied along the long axis of the cantilever can potentially induce parametric resonance under the right frequency and amplitude conditions. Figure $5 \mathrm{~b}$ is an iteration of the second design route by adding a double beam between the anchor and the otherwise anchored end of the cantilever. This additional initial spring structure acts as the electrically undamped additional mechanical amplifier 


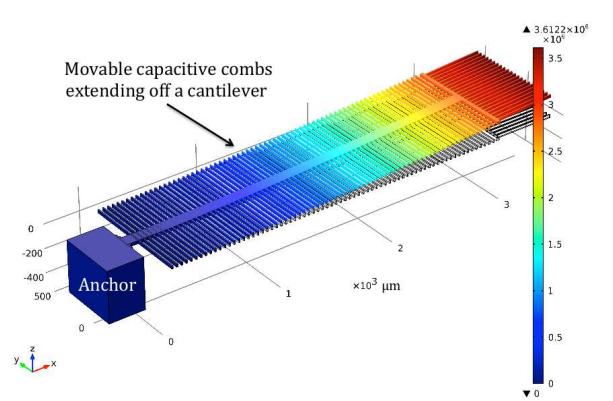

(a) Micro-cantilever beam designed as part of an electrostatic transducer.

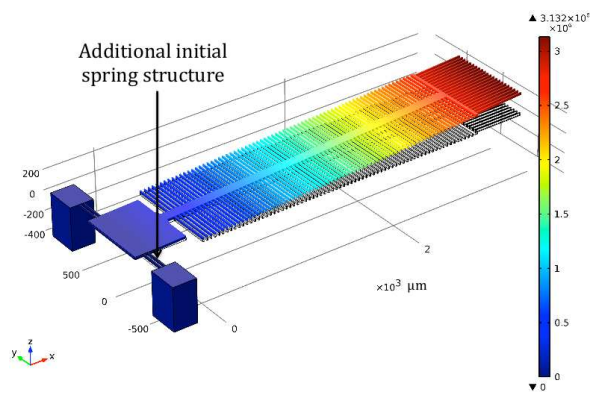

(b) Initial springs added to act as design aids to passively minimise and overcome the initiation threshold amplitude.

Figure 5: Design models of the MEMS cantilevers in COMSOL Multiphysics. Direct excitation equates to an out-of-plane driving force, whereas an in-plane excitation can potentially induce parametric resonance. Capacitive comb fingers extends off the cantilever beam. Accompanying parallel fixed comb fingers are not shown here.

(direct resonator) and enables the possibility of observing auto-parametric resonance.

Auto-parametric resonance is a subset of parametric resonance, which unlike externally induced parametric resonance, arises from a certain integer ratio relationship between the resonant frequencies of multiple coupled resonating components resulting in self-excitation. In this case, if the in-plane natural frequency of the additional initial spring is twice that of the out-of-plane natural frequency of the suspended cantilever beam; then when the initial spring resonates at its fundamental mode of resonance, parametric resonance can be internally induced in the cantilever. Therefore, this establishes auto-parametric resonance. Mathematically, both subsets of parametric resonance are defined by the same equations.

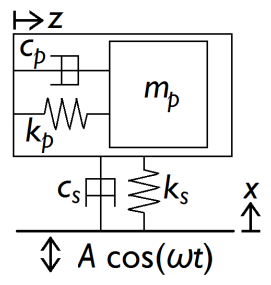

Figure 6: Mass-spring-damper equivalent of the threshold-aided parametrically excited system.

The threshold-aided parametric harvester design can be reduced to a second order mass-spring-damper system illustrated in figure 6 ; where, $c_{s}, k_{s}, c_{p}, k_{p}, m_{p}, x$ and $z$ are damping of the additional initial double beam spring, stiffness of the initial spring, damping of the cantilever spring, stiffness of the cantilever spring, effectiveness mass of the system, displacement of the initial spring and displacement of the cantilever spring respectively. The initial spring is able to pass along energy absorbed from the mechanical excitation to the subsidiary cantilever spring. However, as mentioned already, $z$ must possess an initial displacement for any orthogonal propagation of vibration to take place. 


\subsection{Prototypes}

The designs were fabricated using the MEMSCAP foundry Silicon-On-Insulator (SOI) Multi-User MEMS Processes (MUMPs) and sample devices can be seen in figure 7 . The process allows a device silicon thickness of either $25 \mu \mathrm{m}$ or $10 \mu \mathrm{m}$, as well as metal contact layers.

Three designs were fabricated as listed below and the volume values include the anchors with metal pads,

- Purely parametric harvester (small) with $1000 \mu \mathrm{m}$ in beam length (figure 7a)

- $25 \mu \mathrm{m}$ device volume: $\sim 0.0998 \mathrm{~mm}^{3}$

- $10 \mu \mathrm{m}$ device volume: $\sim 0.0785 \mathrm{~mm}^{3}$

- Purely parametric harvester with $2000 \mu \mathrm{m}$ in beam length (figure $7 \mathrm{~b}$ )

$-25 \mu \mathrm{m}$ device volume: $\sim 0.121 \mathrm{~mm}^{3}$

- $10 \mu \mathrm{m}$ device volume: $\sim 0.0875 \mathrm{~mm}^{3}$

- Threshold-aided parametric harvester with $2000 \mu \mathrm{m}$ in beam length (figure 7c)

- $25 \mu \mathrm{m}$ device volume: $\sim 0.147 \mathrm{~mm}^{3}$

- $10 \mu \mathrm{m}$ device volume: $\sim 0.105 \mathrm{~mm}^{3}$
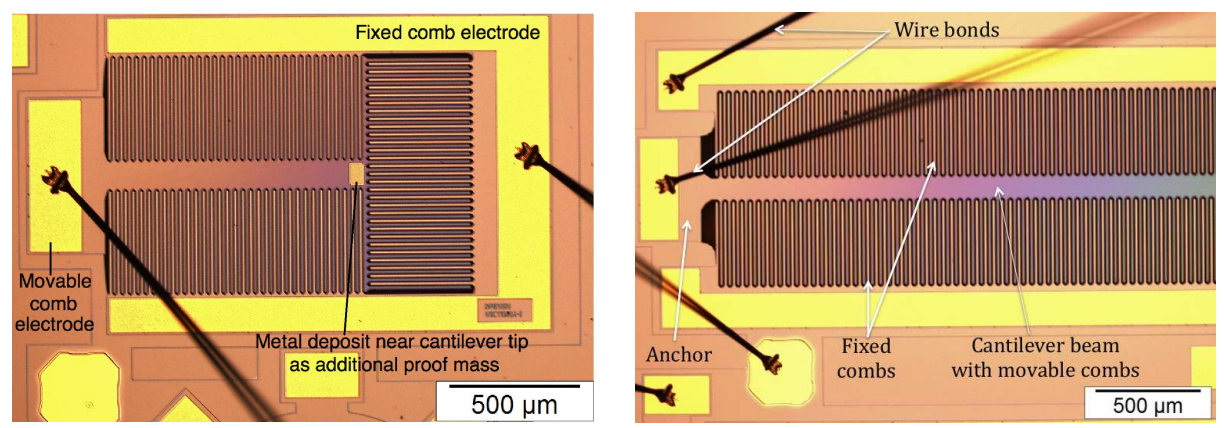

(a) A smaller prototype of the parametrically excited cantilever-based vibration harvester.

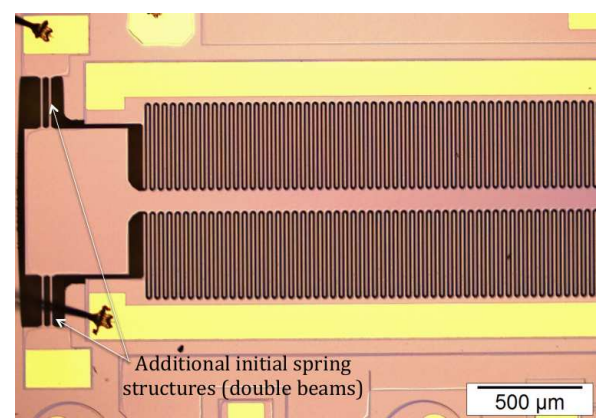

(b) Parametrically excited cantilever-based vibration harvester.

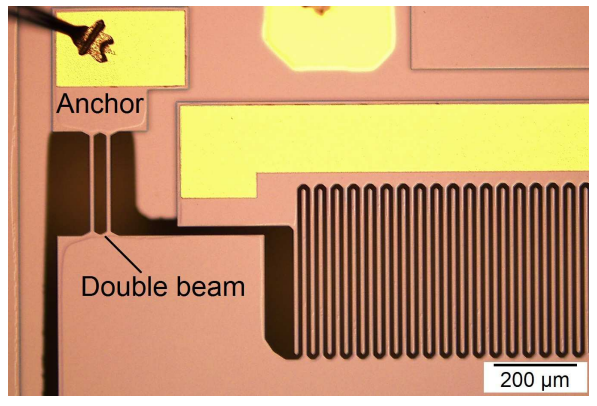

(c) Threshold aided and potentially auto-

(d) The initial spring (double beam) acts as the parametric harvester. threshold-aid, amplifying base excitation.

Figure 7: SOIMUMPs MEMS realisation of three prototype designs at both $25 \mu \mathrm{m}$ and $10 \mu \mathrm{m}$ wafer thickness.

The first two prototypes are essentially smaller and larger versions of a cantilever beam. For each movable comb finger (10 $\mu \mathrm{m}$ wide and $400 \mu \mathrm{m}$ long) extending from the 
cantilever beam, a corresponding fixed comb finger of the same dimensions is placed beside it with $10 \mu \mathrm{m}$ gap spacing. Therefore, a parallel capacitive plate configuration is established throughout the comb finger structures. The third prototype incorporates double beams as the initial springs. The entire shuttle is only anchored through the sets of double beams on either sides.

The decision of employing an electrostatic transducer rather than a more effective transduction mechanism such as piezoelectric [4] is purely due to the fabrication constraints imposed by the foundry process. Maximising absolute power output, although desired, is not the most critical factor since the focal point of the investigation here is on analysing the effectiveness of the resonant behaviour of the mechanical mechanism.

\subsection{Resonant modes}

Various COMSOL simulated mode shapes of the threshold-aided parametric harvester design are shown in figure 8. Equivalent sets of mode shapes for the cantilever-based purely parametric harvester were also simulated but not shown here.

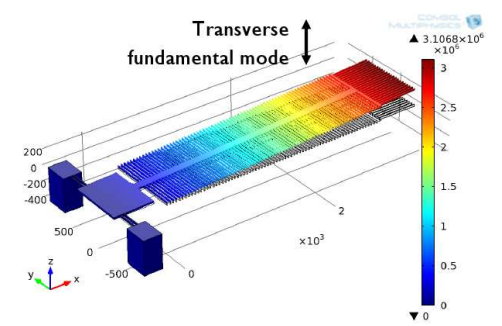

(a) Cantilever 1st transverse at $763.8 \mathrm{~Hz}$

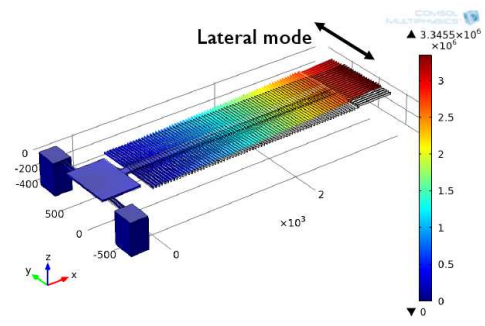

(c) Cantilever 1st lateral at $4569.6 \mathrm{~Hz}$

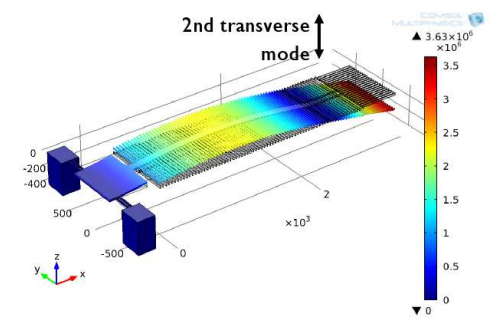

(e) Cantilever 2nd transverse at $6402.3 \mathrm{~Hz}$

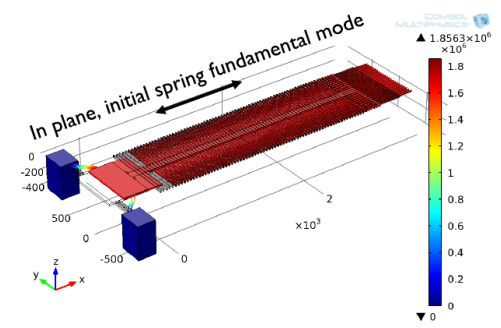

(b) Initial spring 1st in-plane at $18,260.1 \mathrm{~Hz}$

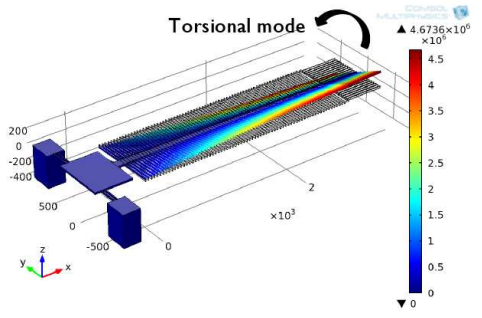

(d) Cantilever 1st torsional at 12,220.3 Hz

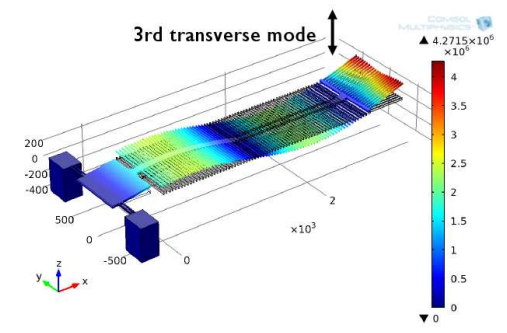

(f) Cantilever 3rd transverse at 19,125.5 Hz

Figure 8: COMSOL eigenfrequency simulation of various resonant mode shapes of the threshold-aided design with $25 \mu \mathrm{m}$ thick silicon device layer. Colour bar represents displacement, which relates to the electrical output of electrostatic transducers. 
The cantilever beam displaces with an out-of-plane gap-overlapping electrostatic motion for the fundamental mode of resonance as illustrated in figure 8a. Parametric resonant modes, regardless of the order, have identical frequency and mode shape response as the out-of-plane fundamental mode shown here. This mode shape is synonymous with the purely parametric harvester.

The in-plane gap-closing mode figure 8b arises from the fundamental mode of the initial springs under direct excitation. This mode is absent from the purely parametric harvesters due to the lack of this additional degree-of-freedom. Therefore, the electrostatic gap-closing motion is also absent from the sole cantilever design.

Other higher transverse modes as well as lateral and torsional modes were present for both threshold-aided and purely parametric harvester designs. However, for an electrostatic transducer, torsional and higher transverse modes are not expected to be observed due to charge neutralisation from their particular motions. The lateral mode refers to an in-plane gap-overlapping configuration similar to one of the mechanisms defined by [2].

\subsection{Device thickness}

Multiple specimens for all three prototype designs, both at $25 \mu \mathrm{m}$ and $10 \mu \mathrm{m}$ thickness, were fabricated and studied. Although the $10 \mu \mathrm{m}$ thickness significantly sacrificed the surface area of the capacitive plate, the overall natural frequency of the device can be minimised for realistic vibration levels. Metal deposits are also added to the free end of the cantilever as shown in figure $7 \mathrm{a}$ to further minimise the resonant frequencies.

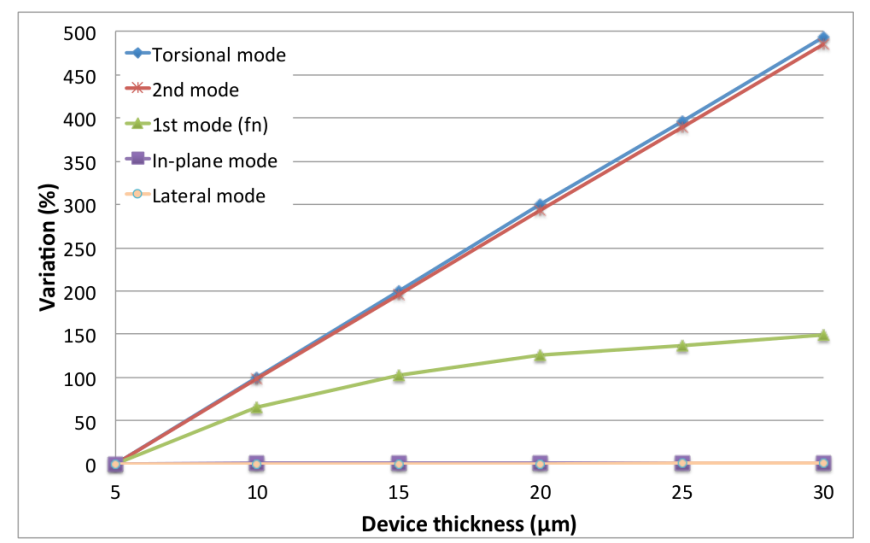

Figure 9: COMSOL simulation of varying device thickness and its correlation with the frequencies of various resonant modes.

COMSOL simulation of the threshold-aided design was carried out to characterise the correlation of device thickness and resonant frequencies of various resonant modes. Figure 9 illustrates a summary of the simulation results for the threshold-aided design at different thickness. The vertical axis denotes the percentage variation of the parameters of interest with device thickness of $5 \mu \mathrm{m}$ as the initial reference. The in-plane fundamental mode of initial spring and the lateral mode of the cantilever beam were not significantly influenced by thickness. This is because both these modes displace in the directions of the length and width of the structure. On the other 
hand, all transverse and torsional modes either entirely or partially involve out-ofplane motion; therefore, are dependent on the thickness of the structure. The general frequency trend is a steady decrease with diminishing thickness.

\section{Results and discussion}

\subsection{Apparatus}

Experimental tests were carried out using the mechanical shaker set up shown in figure 10. The shaker was driven by a function generator via a power amplifier. A commercial three axes accelerometer was mounted alongside the vibrating set up to measure the induced mechanical acceleration by the shaker. The measurement circuit employed for the electrostatic harvester was based on a standard voltage follower configuration with a unity gain op-amp [11]. All tests were undertaken at normal atmospheric pressure and and room temperature. The cantilever was mounted with its free end upright in order to overcome the non-zero initial displacement criterion. This arrangement is equivalent to an inverted pendulum, which places the cantilever tip resting in an unstable equilibrium.

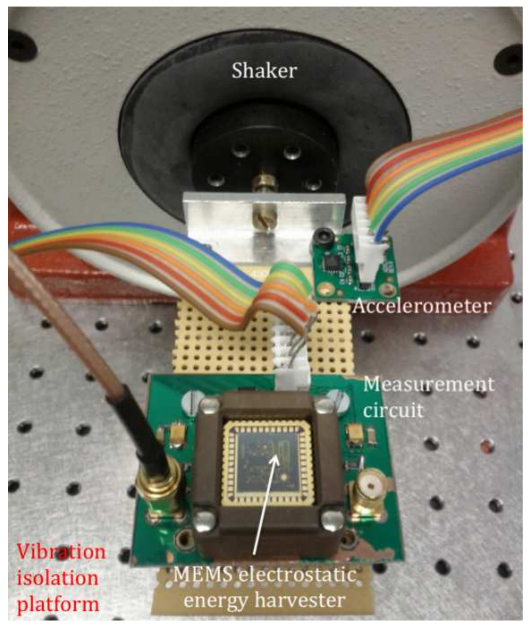

Figure 10: Experimental set up.

\subsection{Measurement methodology}

COMSOL frequency domain simulation of the $25 \mu \mathrm{m}$ thick threshold-aided design (figure $5 \mathrm{~b}$ ) is presented in figure 11a and a frequency sweep by a PolyTec laser vibrometer of the real experimental device (figure 7c) is shown in figure 11b. These frequency domain plots revealed no harmonics or other resonant modes at either twice or half of the natural frequencies. Therefore, the possible presence of other directly excited resonant peaks within the vicinity of these frequency ranges can be excluded.

The COMSOL simulation in figure 11a had a relative tolerance of 0.9 and 745,530 degrees of freedom were solved. The figure $11 \mathrm{~b}$ vibrometer scan was carried out with 10,000 times optical magnification, driven by periodic chirp of $0.5 \mathrm{~V}$ and $1 \mathrm{~V}$ DC offset, resolution of 6400 FFT lines, complex of 100 and rectangle windowing function. 


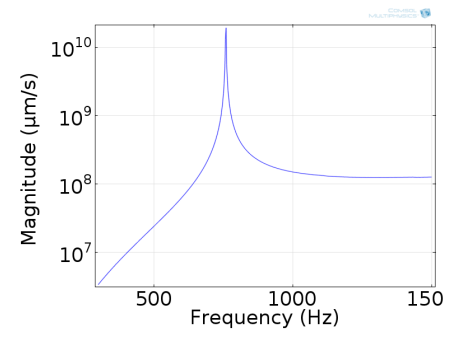

(a) COMSOL frequency sweep, $f_{n}=763.8 \mathrm{~Hz}$

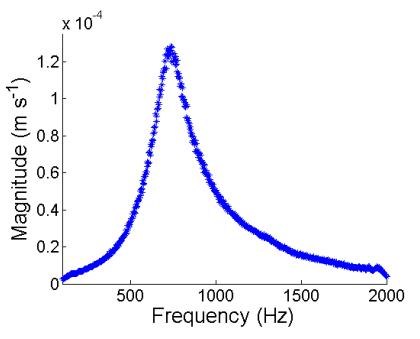

(b) Laser vibrometer scan, $f_{n}=737.5 \mathrm{~Hz}$

Figure 11: Frequency sweeps showing no harmonics or other resonant modes at twice or half of the natural frequency of the threshold-aided prototype. Experimentally measured frequency shifted by $3.44 \%$, potentially a result of MEMS fabrication tolerance and environmental impurities.

The absence of the anticipated parametric resonance in the COMSOL simulation is due to the lack of this resonant phenomenon in the physics of the simulation tool. The reason why vibrometer frequency sweeps, both sine wave sweeps and periodic chirps, also failed to reveal the parametric modes is due to the long transient state required for this resonant mode to build up.

The method employed to experimentally observe the parametric resonant peaks from mechanical excitation was to manually sweep and hold at each frequency point over a period of time for the potential oscillatory build up to take place. Once the parametric resonant regime is activated, varying frequencies within the resonant region will not have to undergo the prolonged build up time to sustain the resonance.

\subsection{Power and threshold}

A summary of experimentally recorded power peaks is presented in table 1 . The first and fourth order parametric resonances were recorded for the $25 \mu \mathrm{m}$ thick threshold-aided parametric harvester at twice and half of the natural frequencies respectively. Although onset of the second order parametric resonance was also observed around natural frequency, steady-state response almost always converged towards the fundamental mode of resonance within the surveyed excitation amplitudes. The third order parametric resonance, theoretically residing at $\frac{2}{3}$ th of the natural frequency, has not yet been experimentally observed. The higher order parametric peaks were not seen for any other prototypes, including the $10 \mu \mathrm{m}$ thick counterpart of the threshold-aided prototype.

As predicted, torsional and other higher transverse modes were not recorded. While the fundamental mode was readily recorded at the respective natural frequencies of each prototype, no significant lateral mode was recorded for any of the prototypes. This could be a result of low lateral capacitive gap-overlap sensitivity for the given excitation amplitude. On the other hand, the in-plane fundamental mode of the initial spring was recorded for both $25 \mu \mathrm{m}$ and $10 \mu \mathrm{m}$ thick threshold-aided prototypes at approximately $18 \mathrm{kHz}$. This result, in contrast to the lateral mode, is in agreement with the higher conversion efficiency of the electrostatic gap-closing mechanism over its in-plane gap-overlapping counterpart as suggested by [2].

For the smaller purely parametric harvester, no parametric resonant peaks were 
Table 1: Experimental power peaks, frequencies, excitation and initiation threshold for various resonant modes of prototypes I, II and III denoting the small purely parametric, larger purely parametric and threshold-aided designs respectively. The term $n / r$ means not recorded and $n / a$ denotes not applicable for the given prototype.

\begin{tabular}{c|ccc|cc}
\multirow{2}{*}{ Device } & \multicolumn{2}{|c|}{ Power peak $(\mathrm{nW}) ;$ Frequency $(\mathrm{Hz})$} & \multicolumn{2}{c}{ Acceleration $\left(\mathrm{ms}^{-2}\right)$} \\
\cline { 2 - 6 } & 1st mode & 1st parametric & in-plane & excitation & threshold \\
\hline I $25 \mu \mathrm{m}$ & $37.7 ; 9100$ & $\mathrm{n} / \mathrm{r}$ & $\mathrm{n} / \mathrm{a}$ & 33 & $\mathrm{n} / \mathrm{r}$ \\
II $25 \mu \mathrm{m}$ & $68.9 ; 1740$ & $134 ; 3420$ & n/a & 33 & 30 \\
III $25 \mu \mathrm{m}$ & $10.7 ; 700$ & $156 ; 1380$ & $20.1 ; 18,200$ & 4.2 & 1.3 \\
\hline I $10 \mu \mathrm{m}$ & $5.46 ; 3800$ & $\mathrm{n} / \mathrm{r}$ & $\mathrm{n} / \mathrm{a}$ & 50 & $\mathrm{n} / \mathrm{r}$ \\
II $10 \mu \mathrm{m}$ & $8.94 ; 630$ & $23.2 ; 1220$ & n/a & 50 & 46 \\
III $10 \mu \mathrm{m}$ & $2.08 ; 380$ & $17.1 ; 750$ & $3.34 ; 18,180$ & 10 & 6.0 \\
\hline
\end{tabular}

observed within the surveyed excitation range of up to $60 \mathrm{~ms}^{-2}$. Whereas, the larger device with comparable size to the threshold-aided prototype did exhibit principal parametric resonance; however, no higher orders were observed. The initiation thresholds of the two prototypes where parametric resonance were recorded are listed below. Over an order of magnitude decrease in this activation barrier can be observed with the addition of the initial spring for the comparable cantilever prototypes.

- Purely parametric harvester initiation threshold:

$-25 \mu \mathrm{m}$ 1st order: $30 \mathrm{~ms}^{-2}$

- $10 \mu \mathrm{m}$ 1st order: $46 \mathrm{~ms}^{-2}$

- Threshold-aided parametric harvester initiation threshold:

$-25 \mu \mathrm{m}$ 1st order: $1.3 \mathrm{~ms}^{-2}$

- $25 \mu \mathrm{m}$ 4th order: $3.3 \mathrm{~ms}^{-2}$

$-10 \mu \mathrm{m}$ 1st order: $6.0 \mathrm{~ms}^{-2}$

The directly excited fundamental mode of resonance is always responsive to excitation even at low amplitudes while parametric resonance has zero response below a certain amplitude threshold. As soon as parametric resonance is activated beyond this activation barrier, its power output rapidly outperforms the direct excitation for the same device. Figure 12 illustrates this relationship for the $25 \mu \mathrm{m}$ thresholdaided prototype, from which the best power performance amongst the experimental prototypes was recorded. The diminishing return at higher amplitudes seen from the levelling off of the graph suggest the presence of higher amplitude dependent nonlinear damping factors, in electrical and/or mechanical domains, which restrict further growth of the resonant peaks.

The threshold-aided prototypes exhibited over an order of magnitude higher in power peak for parametric resonance over directly excited fundamental mode of resonance for acceleration below $1 \mathrm{~g}$ as shown in figure 13. For the $25 \mu \mathrm{m}$ device, at $4.2 \mathrm{~ms}^{-2}$ of excitation, power peaks of $10.7 \mathrm{nW}, 156 \mathrm{nW}$ and $127 \mathrm{nW}$ were recorded for the fundamental mode, first order parametric and fourth order parametric resonances respectively. For the $10 \mu \mathrm{m}$ device, at $1 \mathrm{~g}$ of acceleration, the peak power for principal parametric resonance was $17.1 \mathrm{nW}$ while the fundamental mode produced $2.08 \mathrm{nW}$.

Qualitatively, the frequency domain power response of the purely parametric harvester is similar to that of the threshold-aided harvester. However, since the onset of parametric resonance requires an excess of $3 \mathrm{~g}$ of impact acceleration, the 


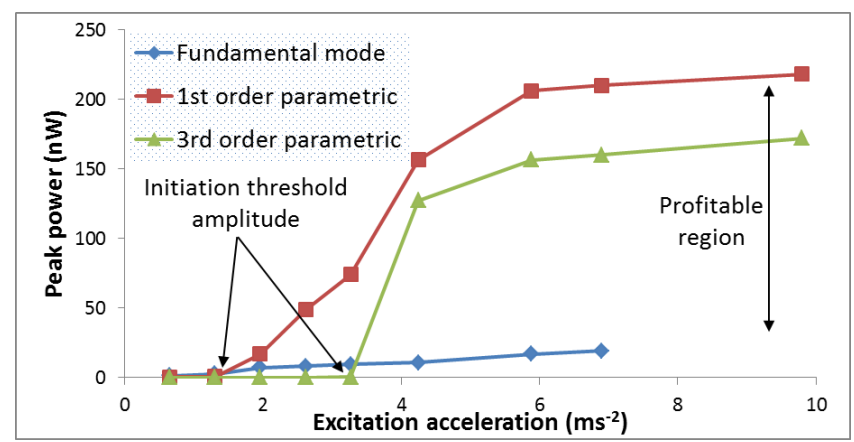

Figure 12: Experimental power response of the $25 \mu \mathrm{m}$ threshold-aided parametric harvester at various resonant regimes plotted against input acceleration.

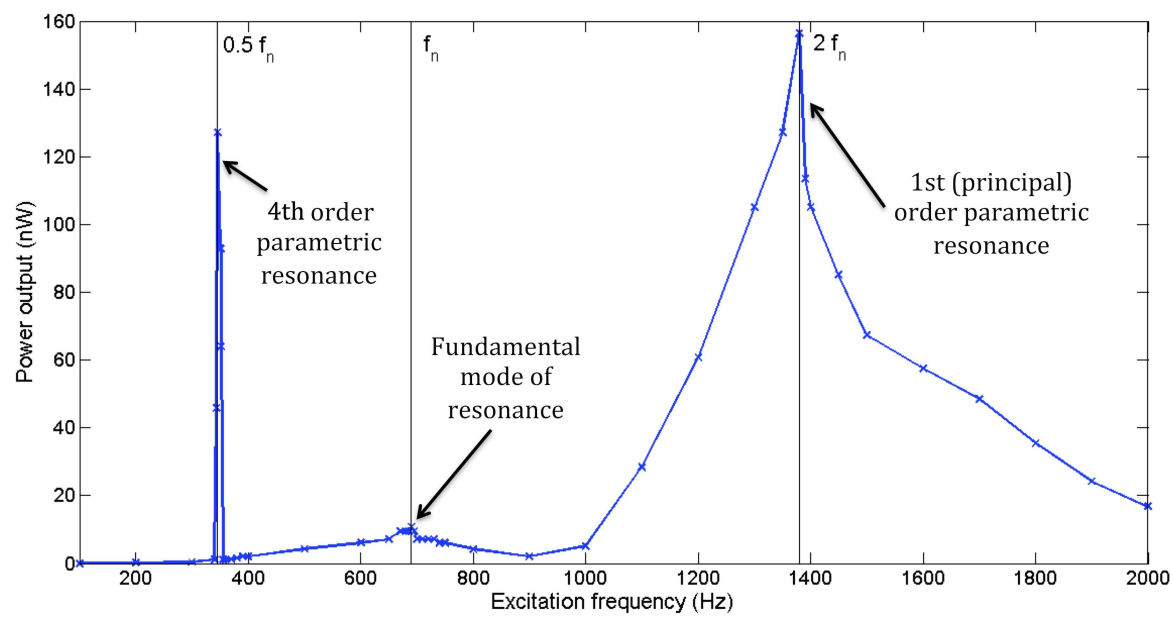

Figure 13: Experimental power response of the $25 \mu \mathrm{m}$ threshold-aided parametric harvester for an input acceleration of $4.2 \mathrm{~ms}^{-2}$. First and fourth order parametric resonances can be observed at twice and half of the natural frequency $f_{n}$ respectively. The onset of second order parametric resonance was observed in the vicinity of $f_{n}$, appearing alternatively to the direct resonant response. However, within the surveyed amplitudes, the steady-state solution at $f_{n}$ always converged towards direct resonance. The third order is yet to be observed.

performance enhancement over its directly excited counterpart is less rewarding and unrealistic for real infrastructural applications.

Table 2 briefly contrasts the results of the $25 \mu \mathrm{m}$ threshold-aided prototype with a few selected electrostatic harvesters in the literature. The figure of merit used for comparison is calculated from power density normalised against the square of input acceleration. The parametric resonant output performs favourably while the direct resonant output performs an order of magnitude worse. Since the compared devices from the literature were all based on direct resonance, the relatively poor performance of the direct resonant output of the prototype suggested room for device optimisation that can potentially further the power performance of parametric resonant output 
even more.

Table 2: Comparing the $25 \mu \mathrm{m}$ threshold-aided parametric harvester results with selected electrostatic counterparts from the literature in terms of power density normalised against acceleration squared.

\begin{tabular}{cccccc}
\hline Reference & $\begin{array}{c}\text { Vol. } \\
\left(\mathrm{mm}^{3}\right)\end{array}$ & $\begin{array}{c}\text { Power } \\
(\mu \mathrm{W})\end{array}$ & $\begin{array}{c}\text { Acc. } \\
\left(\mathrm{ms}^{-2}\right)\end{array}$ & $\begin{array}{c}\text { Freq. } \\
(\mathrm{Hz})\end{array}$ & $\begin{array}{c}\text { Index } \\
\left(\mu \mathrm{Wcm}^{-3} \mathrm{~m}^{-2} \mathrm{~s}^{4}\right)\end{array}$ \\
\hline Parametric (1st order) & 0.147 & 0.156 & 4.2 & 1380 & $\mathbf{6 0 . 2}$ \\
Parametric (4th order) & 0.147 & 0.127 & 4.2 & 342.5 & $\mathbf{4 9 . 0}$ \\
Despesse et al. $(2005)[12]$ & 32.4 & 70 & 9.2 & 50 & 25.5 \\
Roundy et al. $(2002)[2]$ & 1000 & 116 & 2.25 & 120 & 22.9 \\
Wong et al. $(2009)[11]$ & 0.319 & 0.017 & 1.76 & 1400 & 17.2 \\
Fundamental mode & 0.147 & 0.011 & 4.2 & 700 & 4.24 \\
Chu et al. $(2005)[13]$ & 19.96 & 32.34 & 40 & 800 & 1.01 \\
\hline
\end{tabular}

\subsection{Operational frequency bandwidth}

In terms of operational frequency bandwidth, approximately twice the half power bandwidth was recorded for the principal parametric peak $(210 \mathrm{~Hz})$ in contrast to the fundamental mode $(100 \mathrm{~Hz})$ from figure 13. Furthermore, if the half power points of the fundamental mode is taken as the cut-off line for the bandwidth, a band of $1400 \mathrm{~Hz}$ is recorded. However, higher orders of parametric resonance, such as the 4th order peak ( $8 \mathrm{~Hz}$ half power band) from figure 13, displayed significantly narrower bandwidth. Nonetheless, the additional peaks can potentially serve as extra power response regions for these supplementary frequency vicinities.

\section{Future work}

Further design iterations, including auto-parametric designs, based on figure 4 are being explored to better address the issues of initiation threshold amplitude and the non-zero initial displacement requirement. Various MEMS, thin/thick-film and macroscale electromagnetic and piezoelectric devices are also being developed to investigate the power efficiency of this novel technique in contrast to directly excited harvesters when subjected to ambient vibrations in real scenarios.

\section{Conclusion}

This paper presents a novel study of incorporating parametric resonance as a means of mechanical amplification to maximise the electrical power output of MEMS vibration energy harvesters. While this resonant technique theoretically promises better power and frequency response in contrast to the conventional directly excited harvesters, a list of strict initiation criteria, such as a minimum excitation amplitude threshold, needs to be fulfilled. A passive design approach to address this limitation has experimentally shown a reduction of the initiation threshold by approximately a factor of 23. Over 14 fold higher power peak and approximately twice the half power band have been recorded for the parametrically excited scenario in contrast to the 
same harvester driven at fundamental mode of resonance. The results also compare favourably with the literature.

\section{References}

[1] C. Kompis and S. Aliwell. Energy harvesting technologies to enable remote and wireless sensing. Technical report, Sens Instr, KTN, June 2008.

[2] S. Roundy, P.K. Wright, and K.S.J. Pister. Micro-electrostatic vibration-to-electricity converters. In Proceedings of IMECE, page 10pp, 2002.

[3] S.P. Beeby, M.J. Tudor, and N.M. White. Energy harvesting vibration sources for microsystems applications. Meas. Sci. Technol., 17(12):R175-R195, 2006.

[4] S. Priya and D.J. Inman. Energy Harvsting Technologies. Springer US, New York, 2009.

[5] P.D. Mitcheson, E.M. Yeatman, G.K. Rao, A.S. Holmes, and T.C. Green. Energy harvesting from human and machine motion for wireless electronic devices. Proc. IEEE, 96(9):1457$1486,2008$.

[6] E.I. Butikov. Parametric resonance in a linear oscillator at square-wave modulation. Eur. J. Phys., 26(1):157-174, 2005.

[7] M.F. Daqaq, C. Stabler, Y. Qaroush, and T. Seuaciuc-Osorio. Investigation of power harvesting via parametric excitations. J. Intel. Mat. Syst. Str., 20(5):545-557, 2009.

[8] Nicholas Minorsky. Nonlinear Oscillations. Robert E. Krieger, New York, 1974.

[9] M. Sharma, E.H. Sarraf, and E. Cretu. Parametric amplification/damping in mems gyroscopes. In IEEE MEMS, pages 617-620, 17 March 2011.

[10] Y. Jia, J. Yan, K. Soga, and A.A. Seshia. A parametrically excited vibration energy harvester. J. Intel. Mat. Syst. Str., 2013. first online 18 June 2013, doi: 10.1177/1045389X13491637.

[11] Z.J. Wong, J. Yan, K. Soga, and A.A. Seshia. A multi-degree-of-freedom electrostatic mems power harvester. In PowerMEMS, pages 300-303, Washington, 1-4 December 2009.

[12] G. Despesse, T. Jager, J. Chaillout, J. Leger, A. Vassilev, S. Basrour, and B. Chalot. Fabrication and characterisation of high damping electrostatic micro devices for vibration energy scavenging. In Proc. Design, Test, Integration and Packaging of MEMS and MOEMS, pages 386-390, 2005.

[13] Y.S. Chu, C.T. Kuo, and Y. Chiu. A mems electrostatic vibration-to-electricity energy converter. In PowerMEMS, pages 49-52, Tokyo, 28-30 November 2005. 